\title{
Hypoxemia in the ICU: prevalence, treatment, and outcome
}

\author{
SRLF Trial Group*
}

\begin{abstract}
Background: Information is limited regarding the prevalence, management, and outcome of hypoxemia among intensive care unit (ICU) patients. We assessed the prevalence and severity of hypoxemia in ICU patients and analyzed the management and outcomes of hypoxemic patients.

Methods: This is a multinational, multicenter, 1-day point prevalence study in 117 ICUs during the spring of 2016. All patients hospitalized in an ICU on the day of the study could be enrolled. Hypoxemia was defined as a $\mathrm{PaO}_{2} / \mathrm{FiO}_{2}$ ratio $\leq 300 \mathrm{mmHg}$ and classified as mild $\left(\mathrm{PaO}_{2} / \mathrm{FiO}_{2}\right.$ between 300 and 201), moderate $\left(\mathrm{PaO}_{2} / \mathrm{FiO}_{2}\right.$ between 200 and 101), and severe $\left(\mathrm{PaO}_{2} / \mathrm{FiO}_{2} \leq 100 \mathrm{mmHg}\right)$.

Results: Of 1604 patients included, 859 (54\%, 95\% Cl 51-56\%) were hypoxemic, 51\% with mild ( $n=440)$, 40\% with moderate $(n=345)$, and $9 \%(n=74)$ with severe hypoxemia. Among hypoxemic patients, $61 \%(n=525)$ were treated with invasive ventilation, $10 \%(n=84)$ with non-invasive ventilation, $5 \%(n=45)$ with high-flow oxygen therapy, $22 \%$ $(n=191)$ with standard oxygen, and $1.6 \%(n=14)$ did not receive oxygen. Protective ventilation was widely used in invasively ventilated patients. Twenty-one percent of hypoxemic patients $(n=178)$ met criteria for acute respiratory distress syndrome (ARDS) including 65 patients (37\%) with mild, 82 (46\%) with moderate, and 31 (17\%) with severe ARDS. ICU mortality was $27 \%$ in hypoxemic patients and significantly differed according to severity: $21 \%$ in mild, $26 \%$ in moderate, and $50 \%$ in patients with severe hypoxemia, $p<0.001$. Multivariate Cox regression identified moderate and severe hypoxemia as independent factors of ICU mortality compared to mild hypoxemia (adjusted hazard ratio 1.38 [1.00-1.90] and 2.65 [1.69-4.15], respectively).
\end{abstract}

Conclusions: Hypoxemia affected more than half of ICU patients in this 1-day point prevalence study, but only $21 \%$ of patients had ARDS criteria. Severity of hypoxemia was an independent risk factor of mortality among hypoxemic patients.

Trial registration NCT 02722031

Keywords: Hypoxemia, Epidemiology, Critical care, ARDS-acute respiratory failure, Invasive ventilation

\section{Background}

Hypoxemia is frequent and potentially life-threatening in critically ill patients. However, the prevalence, management, and outcomes of hypoxemia in non-selected intensive care unit (ICU) patients are poorly known. Most epidemiological studies have specifically focused on patients under mechanical ventilation [1-3] or those with criteria for acute respiratory distress syndrome

\footnotetext{
*Correspondence: david.grimaldi@erasme.ulb.ac.be
} Brussels, Belgium
(ARDS) [4-9], and none covered the entire spectrum of the whole hypoxemic patients. Whereas mechanical ventilation may be needed in non-hypoxemic patients, a number of hypoxemic patients are treated with oxygen while breathing spontaneously. The proportion of hypoxemic patients treated with standard or high-flow oxygen therapy and without need for mechanical ventilation is unknown among the whole patients in the ICU. Moreover, whereas the severity of hypoxemia was directly associated with mortality in patients with ARDS [10], this relation is not demonstrated among the other hypoxemic patients, especially in non-intubated patients. Finally, the 
relationship between respiratory support, hypoxemia severity, and outcome is not straightforward. It appears then important to provide epidemiological data regarding these gaps of knowledge that could help to design future trials on acute respiratory failure.

Therefore, we designed a large, multicenter, 1-day point prevalence study to measure the prevalence of hypoxemia (defined as a $\mathrm{PaO}_{2} / \mathrm{FiO}_{2}$ ratio $\leq 300 \mathrm{mmHg}$ ) among all ICU patients regardless of their oxygenation device and to stratify them according to the severity of hypoxemia $\left(\mathrm{PaO}_{2} / \mathrm{FiO}_{2}\right.$ between 300 and 201 in mild, between 200 and 101 in moderate, and below or equal to $100 \mathrm{mmHg}$ in severe). We also analyzed the potential impact of hypoxemia and its severity on outcome.

\section{Patients and methods}

\section{Study design}

The SPECTRUM study was a 1-day point prevalence study, endorsed by the French Intensive Care Society (Société de Réanimation de Langue Française: www.srlf. org) and conducted during the spring of 2016 in 117 ICUs in 7 French-speaking countries (62 general ICUs, 36 medical ICUs, 19 surgical ICUs). Additional file 1: Table S1 lists the participating centers and their characteristics. All patients already hospitalized in a participating ICU or newly admitted the day of the study could be enrolled. Exclusion criteria were refusal to participate and in France, being under guardianship, without social insurance, or being pregnant. Approval by the ethics committees according to the laws of each participating country was obtained (CE SRLF 15-35). In France, EC waived from written informed consent, and according to French law, the absence of opposition was obtained from patients or a next of kin before study enrollment.

\section{Definitions}

Hypoxemia was defined as a $\mathrm{PaO}_{2} / \mathrm{FiO}_{2}$ ratio of $300 \mathrm{mmHg}$ or less [5]. We stratified hypoxemia severity as mild for $\mathrm{PaO}_{2} / \mathrm{FiO}_{2}$ between 300 and $201 \mathrm{mmHg}$, moderate for $\mathrm{PaO}_{2} / \mathrm{FiO}_{2}$ between 200 and $101 \mathrm{mmHg}$, and severe for $\mathrm{PaO}_{2} / \mathrm{FiO}_{2}$ below or equal to $100 \mathrm{mmHg}$. ARDS was defined according to the Berlin definition [10]. Each single criterion of the ARDS definition was collected separately in the case report form. Patients fulfilling all the criteria were classified as having ARDS. Potential causes of ARDS were collected independently of the presence of hypoxemia and ARDS. Other causes/ mechanisms of hypoxemia were also collected.

Oxygenation administration was classified as: no oxygen supply (ambient air), low-flow (conventional) oxygen whatever the device used (nasal cannula, mask, tracheostomy), high-flow oxygen through nasal cannula, noninvasive ventilation (NIV) whatever the modalities of ventilation, invasive ventilation through tracheal intubation or tracheostomy, and extracorporeal oxygenation. Patients with intermittent modes were classified with the mode in use at the time of data collection.

\section{Data collection}

All data were assessed at the time of arterial blood gas measurement during the morning round. In cases where no arterial blood was drawn, data were recorded when the patient had an $\mathrm{SpO}_{2} \leq 97 \%$. Follow-up was restricted to ICU stay and censored at day 90 after the study day.

\section{$\mathrm{PaO}_{2} / \mathrm{FiO}_{2}$ determination}

$\mathrm{PaO}_{2}$ and $\mathrm{FiO}_{2}$ were recorded simultaneously. In the case of low-flow oxygen without an $\mathrm{FiO}_{2}$ setting device, we calculated $\mathrm{FiO}_{2}$ as $0.21+$ (oxygen flow rate in liters per minute $\times 0.03$ ) [11]. In the absence of $\mathrm{PaO}_{2}$ measurement, we estimated the $\mathrm{PaO}_{2} / \mathrm{FiO}_{2}$ ratio through the $\mathrm{SpO}_{2} / \mathrm{FiO}_{2}$ ratio using the equation proposed by Rice et al. [12], using a $\mathrm{SpO}_{2} \leq 97 \%$ because the Rice equation cannot infer $\mathrm{PaO}_{2}$ at higher $\mathrm{SaO}_{2}$ given the sigmoid relationship between $\mathrm{SaO}_{2}$ and $\mathrm{PaO}_{2}$.

In mechanically ventilated patients, we measured the following parameters: expired tidal volume $\left(V_{\mathrm{t}}\right)$, measured respiratory rate, set positive end expiratory pressure (PEEP) and plateau pressure and PEEP during an inspiratory and expiratory pause in patients in volume control mode without spontaneous breathing. We calculated the driving pressure by subtracting total PEEP from plateau pressure [13].

\section{Statistical analysis}

Prevalence of hypoxemia was computed using the total number of patients included as the denominator, and the $95 \%$ confidence interval was estimated $(95 \% \mathrm{CI})$. We compared the three groups of hypoxemic patients with different degrees of severity in terms of baseline characteristics, comorbidities, and respiratory conditions. Quantitative variables were described as mean (SD) or median (25th-75th centiles), as appropriate, and compared using Student's $t$ test or the Wilcoxon-MannWhitney test. Qualitative variables were described as counts (\%) and compared using the $\chi^{2}$ or the Fisher exact test, as appropriate. The completeness of the database is indicated in tables. No imputation was used for missing data.

Overall survival was estimated using the Kaplan-Meier method, and survival curves were compared according to the severity of hypoxemia using the log-rank test for categorical variables and the Wald test based on a univariate Cox model for quantitative variables. Among hypoxemic patients, Cox proportional hazards regression was performed to estimate unadjusted hazard ratios and their 
95\% CI. Variables associated with $p$ values $<0.20$ were selected for multivariable analyses. Confounders and interactions were tested in bivariate models. The proportional hazards assumption was assessed statistically using the Schoenfeld residuals test.

All tests were two-sided, and $p$ values $<0.05$ were considered significant. Analyses were conducted using Stata v12.1 (StataCorp LP, College Station, TX, USA).

The study was registered in Clinical trials: NCT 02722031.

\section{Results}

\section{Characteristics of hypoxemic patients}

Of the 1748 patients hospitalized in the participating ICUs the day of the study, 1604 were included. Flowchart of the study is given in Fig. 1. The general characteristics of the patients are reported in Table 1. The day of the study, 859 (54\%, 95\% CI 51-56\%) patients were hypoxemic. Comparison between hypoxemic and non-hypoxemic patients is reported in Additional file 1: Table S2. Among the hypoxemic patients, 440 (51\%) had mild, 345 (40\%) moderate, and 74 (9\%) severe hypoxemia. The causes and mechanisms of hypoxemia are given in Table 2. Pneumonia was the main cause of hypoxemia (53\%), but there were a median of 2 [1-3] causes/ mechanisms of hypoxemia per patient. A total of 178 (21\%) patients fulfilled the criteria for ARDS: 65 (37\%) had mild, 82 (46\%) moderate, and 31 (17\%) severe ARDS. ARDS exclusion criteria among the other 228 hypoxemic patients with bilateral infiltrates are shown in Additional file 1: Table S3. Even though the frequency of ARDS increased with severity of hypoxemia $(p<0.001)$, it represented less than $50 \%$ of severely hypoxemic patients (Table 2).

Of the 1604 patients, 1110 (69\%) had an arterial blood gas analysis (ABG) the day of the study. This proportion reached $87 \%(n=749)$ in hypoxemic patients. ABG revealed that the most severely ill patients had the highest level of $\mathrm{PaCO}_{2}$ and the lowest level of $\mathrm{PaO}_{2}$ (Table 3).

Modalities of oxygen administration are reported in Table 3 . Nearly $40 \%$ of the hypoxemic patients and $25 \%$ of the most severely ill were managed with non-invasive devices. Twenty-five per cent of patients on highflow oxygen were severely hypoxemic, compared with less than $10 \%$ on NIV. Sixteen percent of these patients required later invasive ventilation in their ICU stay whatever the mode of support received the study day (Additional file 1: Figure S1). Causes and radiological finding according to the modalities of oxygen supply are depicted in Table 4. Adjunctive therapies designed to improve oxygenation beyond ventilation were mostly used in the severely hypoxemic patients, the most frequent being neuromuscular blockers, followed by extracorporeal membrane oxygenation, inhaled nitric oxide, and prone positioning (Table 3 ).

\section{Ventilator settings in patients on invasive mechanical ventilation}

Among hypoxemic patients under invasive mechanical ventilation, the measured median $V_{\mathrm{t}}$ was $6.9[6.1-7.9]$

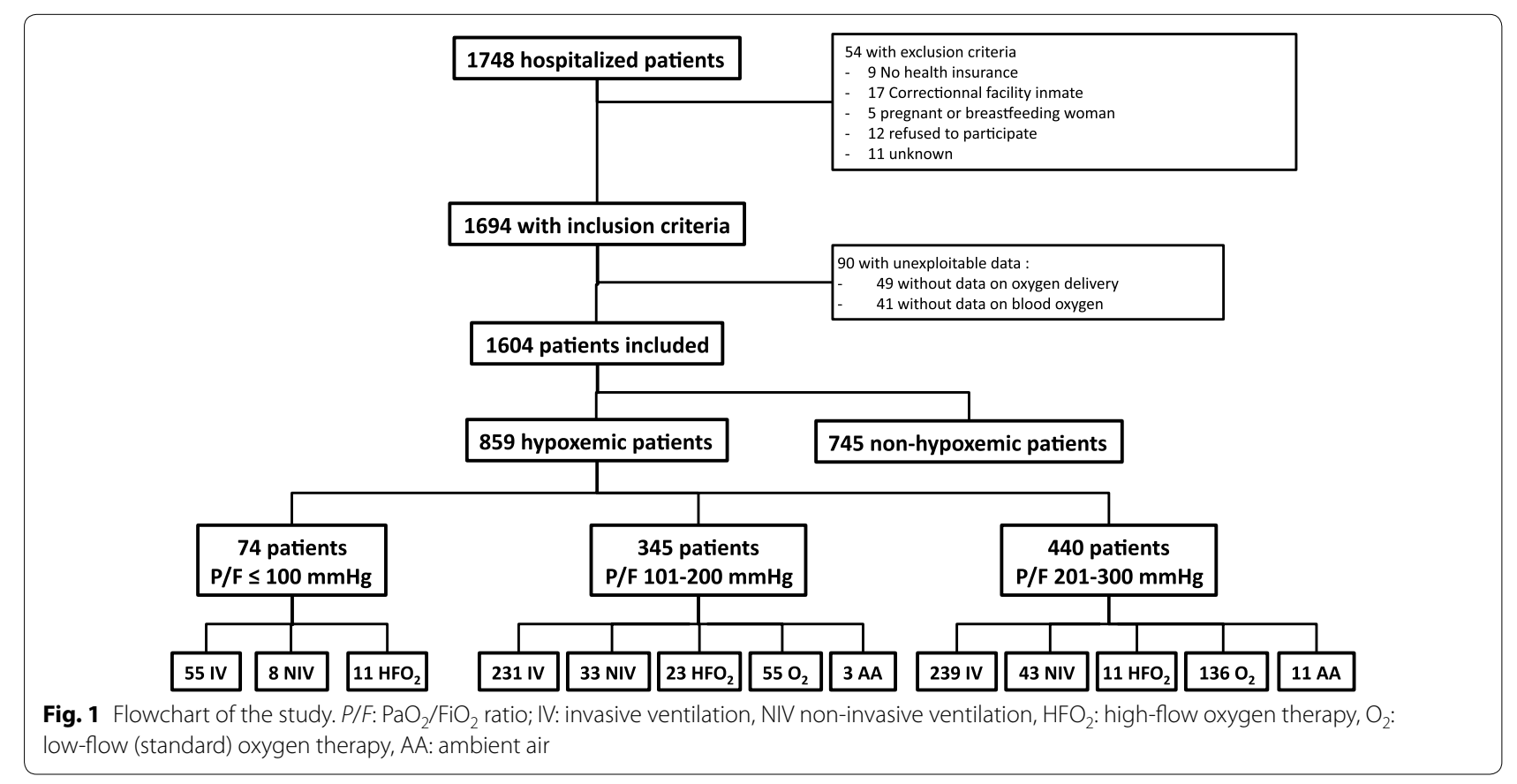


Table 1 Characteristics of hypoxemic patients according to severity of hypoxemia

\begin{tabular}{|c|c|c|c|c|c|}
\hline & $\begin{array}{l}\text { All hypoxemic } \\
\text { patients, } \\
n=859\end{array}$ & $\begin{array}{l}\text { Mild hypoxemia } P / F \\
201-300 \mathrm{mmHg} \text {, } \\
n=440\end{array}$ & $\begin{array}{l}\text { Moderate hypoxemia } P / F \\
101-200 \mathrm{mmHg}, n=345\end{array}$ & $\begin{array}{l}\text { Severe hypoxemia } \\
P / F \leq 100 \mathrm{mmHg}, \\
n=74\end{array}$ & $p$ \\
\hline Age, years median (IQR) & $64[53-73]$ & $65[54-74]$ & $65[53-73]$ & $58[47-69]$ & 0.006 \\
\hline Female, N (\%) & $273(31.8)$ & $142(32.3)$ & $109(31.6)$ & $22(29.7)$ & 0.91 \\
\hline Body mass index $(n=844 / 431 / 341 / 72)$ & $26.1[22.5-31.1]$ & $26.1[22.7-30.9]$ & $26.0[22.5-31.0]$ & $27.0[22.1-34.4]$ & 0.83 \\
\hline \multicolumn{5}{|c|}{ Main diagnosis at ICU admission $(n=858 / 439 / 345 / 74)$} & - \\
\hline Septic shock & $124(14.5)$ & $67(15.2)$ & $49(14.2)$ & $8(10.8)$ & \\
\hline Other shock & $47(5.5)$ & $24(5.4)$ & $17(4.9)$ & $6(8.1)$ & \\
\hline Severe trauma & $20(2.3)$ & $14(3.2)$ & $6(1.7)$ & $0(0)$ & \\
\hline De novo acute respiratory failure & $275(32.0)$ & $118(26.9)$ & $120(34.8)$ & $37(50.0)$ & \\
\hline Acute on chronic respiratory failure & $128(14.9)$ & $55(12.5)$ & $68(19.7)$ & $5(6.8)$ & \\
\hline Coma/seizures & $66(7.7)$ & $43(9.8)$ & $19(5.5)$ & $4(5.4)$ & \\
\hline Metabolic disorders & $18(2.1)$ & $11(2.5)$ & $6(1.7)$ & $1(1.4)$ & \\
\hline Hepatic failure & $6(0.7)$ & $2(0.5)$ & $2(0.6)$ & $2(2.7)$ & \\
\hline Cardiac arrest & $30(3.5)$ & $15(3.4)$ & $12(3.5)$ & $3(4.1)$ & \\
\hline Postoperative surveillance & $96(11.2)$ & $64(14.6)$ & $31(9.0)$ & $1(1.3)$ & \\
\hline Other & $48(5.6)$ & $26(5.9)$ & $15(4.4)$ & $7(9.5)$ & \\
\hline \multicolumn{5}{|l|}{ Admission category $(n=857 / 439 / 344 / 74)$} & 0.004 \\
\hline Medical & $675(78.8)$ & $327(74.5)$ & $281(81.7)$ & $67(90.5)$ & \\
\hline Scheduled surgery & $76(8.9)$ & $49(11.1)$ & $27(7.8)$ & $0(0)$ & \\
\hline Urgent surgery & $92(10.7)$ & $53(12.1)$ & $32(9.3)$ & $7(9.5)$ & \\
\hline Trauma & $14(1.6)$ & $10(2.3)$ & $4(1.2)$ & $0(0)$ & \\
\hline SAPS $\|(n=832 / 425 / 334 / 73)$ & $43[31-57]$ & $42[30-56]$ & $43[31-57]$ & $45[34-61]$ & 0.32 \\
\hline $\begin{array}{l}\text { Chronic respiratory disease (obstructive) } \\
(n=855 / 440 / 342 / 73)\end{array}$ & $288(33.7)$ & $141(32.1)$ & $127(37.1)$ & $20(27.4)$ & 0.16 \\
\hline $\begin{array}{l}\text { Chronic respiratory disease (restrictive) } \\
\quad(n=855 / 440 / 342 / 73)\end{array}$ & $71(8.3)$ & $28(6.4)$ & $37(10.8)$ & $6(8.2)$ & 0.08 \\
\hline $\begin{array}{l}\text { Obstructive sleep apnea syndrome } \\
(n=855 / 440 / 342 / 73)\end{array}$ & $71(8.3)$ & $36(8.2)$ & $29(8.5)$ & $6(8.2)$ & 0.99 \\
\hline $\begin{array}{l}\text { Chronic oxygen therapy } \\
(n=855 / 440 / 342 / 73)\end{array}$ & $71(8.3)$ & $32(7.3)$ & $33(9.7)$ & $6(8.2)$ & 0.49 \\
\hline $\begin{array}{l}\text { Long-term non-invasive ventilation } \\
\qquad(n=855 / 440 / 342 / 73)\end{array}$ & $32(3.7)$ & $17(3.9)$ & $14(4.1)$ & $1(1.4)$ & 0.68 \\
\hline Chronic heart failure $(n=855 / 440 / 342 / 73)$ & $145(17.0)$ & $74(16.8)$ & $62(18.1)$ & $9(12.3)$ & 0.48 \\
\hline Chronic kidney failure $(n=855 / 440 / 342 / 73)$ & $83(9.7)$ & $55(12.5)$ & $23(6.7)$ & $5(6.9)$ & 0.02 \\
\hline Cirrhosis $(n=855 / 440 / 342 / 73)$ & $45(5.3)$ & $25(5.7)$ & $15(4.4)$ & $5(6.9)$ & 0.54 \\
\hline Cancer $(n=855 / 440 / 342 / 73)$ & $79(9.2)$ & $43(9.8)$ & $32(9.4)$ & $4(5.5)$ & 0.50 \\
\hline Immunosuppression ( $n=853 / 439 / 341 / 73)$ & $99(11.6)$ & $46(10.5)$ & $43(12.6)$ & $10(13.7)$ & 0.55 \\
\hline $\begin{array}{l}\text { Withholding/withdrawal of treatment the } \\
\text { day of the study }\end{array}$ & $105(12.2)$ & $41(9.3)$ & 47 (13.6) & $17(23)$ & 0.002 \\
\hline
\end{tabular}

$\mathrm{mL} / \mathrm{kg}$ of ideal body weight and $77 \%$ received a tidal volume of $8 \mathrm{~mL} / \mathrm{kg}$ or less of ideal body weight. Tidal volume decreased with the severity of hypoxemia (Table 3 ). PEEP was set at $6[5-10] \mathrm{cmH}_{2} \mathrm{O}$ in the whole cohort and increased with the severity of hypoxemia to a median of 10 [8-12] $\mathrm{cmH}_{2} \mathrm{O}$ in the severely hypoxemic patients. Whereas plateau pressure increased with severity of hypoxemia, median driving pressure was below 15 $\mathrm{cmH}_{2} \mathrm{O}$ and similar in the 3 groups of patients (Table 3 ).

\section{Outcomes}

Median ICU length of stay was 12 [5-28] days and ICU mortality was $20 \%$ in the 1604 patients of the study. In the hypoxemic population, median ICU length of stay was higher (16 [7-32] vs 8 [3-22] days in non-hypoxemic patients, $p<0.001$ ) but not related to the severity of hypoxemia even when looking only at the patients leaving the ICU alive (Table 3). Patients with invasive 
Table 2 Mechanisms/causes of hypoxemia a and radiological findings according to severity of hypoxemia on the day of the study

\begin{tabular}{|c|c|c|c|c|c|}
\hline & $\begin{array}{l}\text { All hypoxemic } \\
\text { patients, } n=859\end{array}$ & $\begin{array}{l}\text { Mild } \\
\text { hypoxemia, } \\
n=440\end{array}$ & $\begin{array}{l}\text { Moderate } \\
\text { hypoxemia, } \\
n=345\end{array}$ & $\begin{array}{l}\text { Severe } \\
\text { hypoxemia, } \\
n=74\end{array}$ & $p$ \\
\hline Pneumonia, $N(\%)(n=852 / 440 / 339 / 73)$ & $453(53.2)$ & $189(43.0)$ & $204(60.2)$ & $60(82.2)$ & $<0.001$ \\
\hline Aspiration $(n=852 / 440 / 339 / 73)$ & $74(8.7)$ & $30(6.8)$ & $33(9.7)$ & $11(15.1)$ & 0.05 \\
\hline Acute on chronic respiratory failure $(n=853 / 440 / 340 / 73)$ & $166(19.5)$ & $73(16.6)$ & $80(23.5)$ & $13(17.8)$ & 0.05 \\
\hline Cardiogenic pulmonary edema $(n=853 / 440 / 340 / 73)$ & $127(14.9)$ & $64(14.6)$ & $51(15.0)$ & $12(16.4)$ & 0.91 \\
\hline Pulmonary embolism ( $n=853 / 440 / 340 / 73)$ & $26(3.1)$ & $16(3.6)$ & $7(2.1)$ & $3(4.1)$ & 0.37 \\
\hline Thoracic trauma $(n=852 / 440 / 339 / 73)$ & $21(2.5)$ & $9(2.1)$ & $11(3.2)$ & $1(1.4)$ & 0.59 \\
\hline Pleural effusion ( $n=852 / 440 / 339 / 73)$ & $192(22.5)$ & $99(22.5)$ & $77(22.7)$ & $16(21.9)$ & 0.99 \\
\hline Atelectasis $(n=853 / 440 / 340 / 73)$ & $176(20.6)$ & $93(21.1)$ & $72(21.2)$ & $11(15.1)$ & 0.47 \\
\hline Pneumothorax $(n=853 / 440 / 340 / 73)$ & $15(1.8)$ & $6(1.4)$ & $7(2.1)$ & $2(2.7)$ & 0.49 \\
\hline Fluid overload ( $n=853 / 440 / 340 / 73$ ) & $280(32.8)$ & $140(31.8)$ & $115(33.8)$ & $25(34.3)$ & 0.81 \\
\hline Acute pancreatitis $(n=852 / 440 / 339 / 73)$ & $22(2.6)$ & $14(3.2)$ & $6(1.8)$ & $2(2.7)$ & 0.48 \\
\hline Shock, low $\mathrm{PvO}_{2}(n=853 / 440 / 340 / 73)$ & $110(12.9)$ & $42(9.6)$ & $48(14.1)$ & $20(27.4)$ & $<0.001$ \\
\hline Smoke/toxic inhalation $(n=852 / 440 / 339 / 73)$ & $5(0.6)$ & $1(0.2)$ & $3(0.9)$ & $1(1.4)$ & 0.20 \\
\hline Transfusion-related acute lung injury $(n=852 / 440 / 339 / 73)$ & $44(5.2)$ & $32(7.3)$ & $9(2.7)$ & $3(4.1)$ & 0.01 \\
\hline Pulmonary vasculitis $(n=852 / 440 / 339 / 73)$ & $6(0.7)$ & $3(0.7)$ & $2(0.6)$ & $1(1.4)$ & 0.66 \\
\hline Drowning $(n=851 / 440 / 339 / 72)$ & $1(0.1)$ & $1(0.2)$ & $0(0)$ & $0(0)$ & 1 \\
\hline Other $(n=851 / 440 / 338 / 73)$ & $90(10.6)$ & $43(9.8)$ & $42(12.4)$ & $5(6.9)$ & 0.30 \\
\hline Number of causes of hypoxemia $\left(n=853^{b} / 440 / 340 / 73\right)$ & $2[1-3]$ & $2[1-3]$ & $2[1-3]$ & $2[1-3]$ & $<0.001$ \\
\hline Number of causes of hypoxemia $\left(n=853^{b} / 440 / 340 / 73\right)$ & & & & & $<0.001$ \\
\hline None & $107(12.5)$ & $82(18.6)$ & $22(6.5)$ & $3(4.1)$ & \\
\hline 1 & $230(27.0)$ & $110(25.0)$ & $100(29.4)$ & $20(27.4)$ & \\
\hline 2 & $221(25.9)$ & $110(25.0)$ & $93(27.3)$ & $18(24.7)$ & \\
\hline 3 or more & $295(34.6)$ & $138(31.4)$ & $125(36.8)$ & $32(43.8)$ & \\
\hline Radiological infiltrates $(n=845 / 435 / 337 / 73)$ & $583(69.0)$ & $257(59.1)$ & $260(77.2)$ & $66(90.4)$ & $<0.001$ \\
\hline Unilateral $^{C}(n=581 / 255 / 260 / 66)$ & $176(30.3)$ & $85(33.3)$ & $81(31.2)$ & $10(15.2)$ & 0.02 \\
\hline Bilateral $^{\complement}(n=581 / 255 / 260 / 66)$ & $406(69.6)$ & $171(67.1)$ & $179(68.9)$ & $56(84.9)$ & 0.02 \\
\hline ARDS & $178(20.7)$ & $65(14.7)$ & $82(23.8)$ & $31(41.9)$ & $<0.001$ \\
\hline
\end{tabular}

a Multiple causes and mechanisms could be selected

b In 6 patients data on hypoxemia, causes were not recorded

c Among patients with radiological infiltrates

ventilation had the longest stay in the ICU and the highest mortality (Table 4).

ICU mortality was $12 \%$ in non-hypoxemic patients and $27 \%$ in hypoxemic patients $(p<0.001)$. ICU mortality increased with severity of hypoxemia: $21 \%$ in mildly, $29 \%$ in moderately, and $51 \%$ in severely hypoxemic patients $(p<0.001)$ (Table 3 and Fig. 2) and with invasiveness of ventilator support (Table 4). Additional file 1: Table S4 reports the ICU mortality according to ventilator support and hypoxemia class.

\section{Multivariate analysis}

Taking into account the variables associated with ICU mortality in univariate analysis (Additional file 1: Table S5), multivariate analysis using a Cox model confirmed that moderate hypoxemia and severe hypoxemia were independently associated with ICU mortality compared to mild hypoxemia (adjusted hazard ratio 1.38 [1.00-1.90] and 2.65 [1.69-4.15], respectively), as well as other classic variables (Table 5). We performed several sensitivity analyses to strengthen this result (Additional file 1: Table S6): first, including the oxygen support in the analysis, second analyzing only patients with a $\mathrm{PaO}_{2} / \mathrm{FiO}_{2}$ ratio based on actual $\mathrm{PaO}_{2}$ and $\mathrm{FiO}_{2}$, third excluding patients on chronic oxygen therapy or chronic NIV, and fourth excluding patients with therapeutics withholding/withdrawing. These analyses show similar results, although moderate hypoxemia was no longer significantly associated with mortality (Additional file 1: Table S6). Moreover, analyzing the $\mathrm{PaO}_{2} /$ $\mathrm{FiO}_{2}$ ratio as a continuous variable in the same model 
Table 3 Respiratory conditions and outcome according to severity of hypoxemia

\begin{tabular}{|c|c|c|c|c|c|}
\hline & $\begin{array}{l}\text { All hypoxemic } \\
\text { patients, } \\
n=859\end{array}$ & $\begin{array}{l}\text { Mild hypoxemia } P / F \\
201-300 \mathrm{mmHg} \\
n=440\end{array}$ & $\begin{array}{l}\text { Moderate hypoxemia } P / F \\
101-200 \mathrm{mmHg}, n=345\end{array}$ & $\begin{array}{l}\text { Severe hypoxemia } \\
P / F \leq 100 \mathrm{mmHg} \\
n=74\end{array}$ & $p$ \\
\hline Respiratory rate $(n=846 / 432 / 340 / 74)$ & $23[18-27]$ & $22[18-26]$ & 24 [20-28] & $25[20-30]$ & 0.001 \\
\hline Arterial blood gases & $749(87.2)$ & $368(83.6)$ & $311(90.1)$ & 70 (94.6) & 0.004 \\
\hline $\mathrm{pH}(n=745 / 366 / 309 / 70)$ & $7.41[7.36-7.46]$ & $7.42[7.38-7.45]$ & $7.41[7.36-7.46]$ & $7.38[7.29-7.45]$ & 0.007 \\
\hline $\mathrm{PaCO}_{2}(\mathrm{mmHg})(n=749 / 368 / 311 / 70)$ & $41[36-49]$ & $40[35-46]$ & $43[38-55]$ & $45[39-56]$ & 0.001 \\
\hline $\mathrm{PaCO}_{2}>45 \mathrm{mmHg}(n=749 / 368 / 311 / 70)$ & $260(34.7)$ & $96(26.1)$ & $131(42.1)$ & $33(47.1)$ & $<0.001$ \\
\hline $\mathrm{PaO}_{2}(\mathrm{mmHg})(n=749 / 368 / 311 / 70)$ & 78 [66-92] & 84 [73-100] & $74[64-88]$ & $65[56-74]$ & $<0.001$ \\
\hline $\mathrm{FiO}_{2}(\%)(n=749 / 368 / 311 / 70)$ & $0.40[0.33-0.50]$ & $0.35[0.30-0.40]$ & $0.50[0.40-0.60]$ & $1[0.70-1]$ & $<0.001$ \\
\hline $\mathrm{HCO}_{3}-(\mathrm{meq} / \mathrm{L})(n=747 / 368 / 309 / 70)$ & $26[23-30]$ & 26 [22-29] & 27 [23-32] & $26[23-30]$ & 0.03 \\
\hline Lactates (meq/L) $(n=630 / 312 / 257 / 61)$ & $1.3[1.0-1.9]$ & $1.2[0.9-1.7]$ & $1.3[1.0-1.9]$ & $1.4[1.1-2.7]$ & 0.02 \\
\hline Oxygenation modalities, $N(\%)$ & & & & & $<0.001$ \\
\hline Ambient air & $14(1.6)$ & $11(2.5)$ & $3(0.9)$ & $0(0)$ & \\
\hline Low-flow oxygen & $191(22.2)$ & $136(30.9)$ & $55(15.9)$ & $0(0)$ & \\
\hline High-flow oxygen & $45(5.2)$ & $11(2.5)$ & $23(6.7)$ & $11(14.9)$ & \\
\hline Non-invasive ventilation & $84(9.8)$ & $43(9.8)$ & $33(9.6)$ & $8(10.8)$ & \\
\hline Invasive ventilation & $525(61.2)$ & $239(54.3)$ & $231(66.9)$ & $55(74.3)$ & \\
\hline \multicolumn{6}{|l|}{ Ventilator settings $^{\mathrm{a}}$} \\
\hline $\begin{array}{l}\text { Tidal volume, } \mathrm{mL} / \mathrm{kg} \text { of ideal } \\
\text { body weight; median IQR } \\
(n=509 / 230 / 225 / 54)\end{array}$ & $6.9[6.1-7.9]$ & $7.1[6.2-8.1]$ & $6.8[6.0-7.6]$ & $6.1[4.8-6.6]$ & $<0.001$ \\
\hline $\begin{array}{l}\text { TV } \leq 8 \mathrm{~mL} / \mathrm{kg} \text { of ideal body weight } \\
(n=509 / 230 / 225 / 54)\end{array}$ & $393(77.2)$ & $165(71.7)$ & $178(79.1)$ & $50(92.6)$ & 0.003 \\
\hline $\operatorname{PEEP}(\mathrm{cmH} 20)(n=519 / 236 / 229 / 54)$ & $6[5-10]$ & $5[5-8]$ & $7[5-10]$ & $10[8-12]$ & $<0.001$ \\
\hline $\begin{array}{l}\text { Plateau pressure }(\mathrm{cmH} 20) \\
\quad(n=186 / 66 / 84 / 36)\end{array}$ & $22.5[19-27]$ & 20 [16-24] & $23.5[20-28]$ & $25.5[23-29.5]$ & $<0.001$ \\
\hline $\begin{array}{l}\text { Driving pressure }(\mathrm{cmH} 20) \\
\quad(n=186 / 66 / 84 / 36)\end{array}$ & $14[10-18]$ & $13[9-17]$ & $14[11-18]$ & 13 [10-19] & 0.31 \\
\hline \multicolumn{6}{|l|}{ Adjunctive therapies } \\
\hline Prone positioning $(n=516 / 232 / 230 / 54)$ & $22(4.3)$ & $3(1.3)$ & $13(5.7)$ & $6(11.1)$ & 0.001 \\
\hline Inhaled NO ( $n=513 / 233 / 226 / 54)$ & $19(3.7)$ & $3(1.3)$ & $9(4.0)$ & $7(13.0)$ & 0.001 \\
\hline $\begin{array}{l}\text { Continuous intravenous sedation, } N(\%) \\
(n=522 / 237 / 231 / 54)\end{array}$ & $321(61.5)$ & $121(51.1)$ & $159(68.8)$ & $41(75.9)$ & $<0.001$ \\
\hline $\begin{array}{l}\text { Neuromuscular blocking agents, } N(\%) \\
(n=522 / 237 / 231 / 54)\end{array}$ & $83(15.9)$ & $11(4.6)$ & $44(19.1)$ & $28(51.9)$ & $<0.001$ \\
\hline $\begin{array}{l}\text { Extracorporeal oxygenation, } N(\%) \\
\quad(n=522 / 237 / 231 / 54)\end{array}$ & $26(3.0)$ & $5(1.1)$ & $7(2.0)$ & $14(18.9)$ & $<0.001$ \\
\hline $\begin{array}{l}\text { ICU length of stay (days) } \\
\qquad(n=840 / 431 / 336 / 73)\end{array}$ & $16[7-32]$ & 15 [7-32] & $16[8-31.5]$ & $18[7-37]$ & 0.89 \\
\hline $\begin{array}{l}\text { ICU length of stay (days) in ICU survivors } \\
(n=625 / 342 / 246 / 37)\end{array}$ & $15[7-30]$ & $15[7-30]$ & $14[6.5-30]$ & $20.5[9-46.5]$ & 0.27 \\
\hline $\begin{array}{l}\text { ICU mortality, } N(\%) \\
\quad(n=839 / 431 / 335 / 73)\end{array}$ & $225(26.7)$ & $92(21.3)$ & $96(28.5)$ & $37(50.7)$ & $<0.001$ \\
\hline
\end{tabular}

a Invasively ventilated patients $n=525$

b Only in patients in whom plateau pressure was measurable (i.e., patients in VAC without spontaneous breathing). $n=186$

confirmed the independent association between the severity of hypoxemia and mortality (adjusted hazard ratio for each $10 \mathrm{mmHg}$ decrease 1.06 [1.03-1.09], $p<0.001)$.

\section{Discussion}

This large, multicenter study gives for the first time data on the prevalence of hypoxemia in non-selected ICU-hospitalized patients. Strikingly, though more 
Table 4 Mechanisms/causes of hypoxemia and radiological findings according to oxygenation modalities

\begin{tabular}{|c|c|c|c|c|c|c|}
\hline & Ambient air, $n=14$ & $\begin{array}{l}\text { Low-flow } \\
\text { oxygen, } \\
n=191\end{array}$ & $\begin{array}{l}\text { High-flow } \\
\text { oxygen, } \\
n=45\end{array}$ & $\begin{array}{l}\text { Non-invasive } \\
\text { ventilation, } \\
n=84\end{array}$ & $\begin{array}{l}\text { Invasive } \\
\text { ventilation, } \\
n=525\end{array}$ & $p$ \\
\hline Pneumonia, $N(\%)(n=852 / 14 / 189 / 45 / 83 / 521)$ & $3(21.4)$ & $62(32.8)$ & $34(75.6)$ & $37(44.6)$ & $317(60.8)$ & $<0.001$ \\
\hline Aspiration $(n=852 / 14 / 190 / 45 / 83 / 520)$ & $0(0)$ & $11(5.8)$ & $0(0)$ & $3(3.6)$ & $60(11.5)$ & 0.003 \\
\hline $\begin{array}{l}\text { Acute on chronic respiratory failure } \\
\quad(n=853 / 14 / 190 / 45 / 83 / 521)\end{array}$ & $0(0)$ & $32(16.8)$ & $4(8.9)$ & $41(49.4)$ & $89(17.1)$ & $<0.001$ \\
\hline $\begin{array}{l}\text { Cardiogenic pulmonary edema } \\
\quad(n=853 / 14 / 190 / 45 / 83 / 521)\end{array}$ & $2(14.3)$ & $28(14.7)$ & $4(8.9)$ & $15(18.1)$ & $78(15.0)$ & 0.75 \\
\hline Pulmonary embolism ( $n=853 / 14 / 190 / 45 / 83 / 521)$ & $0(0)$ & $5(2.6)$ & $2(4.4)$ & $2(2.4)$ & $17(3.3)$ & 0.93 \\
\hline Thoracic trauma $(n=852 / 14 / 190 / 45 / 83 / 520)$ & $0(0)$ & $5(2.6)$ & $2(4.4)$ & $0(0)$ & $14(2.7)$ & 0.46 \\
\hline Pleural effusion ( $n=852 / 14 / 189 / 45 / 83 / 521)$ & $2(14.3)$ & $34(18.0)$ & $11(24.4)$ & $24(28.9)$ & $121(23.2)$ & 0.3 \\
\hline Atelectasis $(n=853 / 14 / 190 / 45 / 83 / 521)$ & $2(14.3)$ & $30(15.8)$ & $10(22.2)$ & $22(26.5)$ & $112(21.5)$ & 0.27 \\
\hline Pneumothorax $(n=853 / 14 / 190 / 45 / 83 / 521)$ & $0(0)$ & $4(2.1)$ & $0(0)$ & $0(0)$ & $11(2.1)$ & 0.72 \\
\hline Fluid overload $(n=853 / 14 / 190 / 45 / 83 / 521)$ & $2(14.3)$ & $47(24.7)$ & $12(26.7)$ & $28(33.7)$ & $191(36.7)$ & 0.02 \\
\hline Acute pancreatitis ( $n=852 / 14 / 190 / 45 / 83 / 520)$ & $0(0)$ & $6(3.2)$ & $0(0)$ & $1(1.2)$ & $15(2.9)$ & 0.81 \\
\hline Shock, low $\mathrm{PvO}_{2}(n=853 / 14 / 190 / 45 / 83 / 521)$ & $0(0)$ & $6(3.2)$ & $3(6.7)$ & $6(7.2)$ & $95(18.2)$ & $<0.001$ \\
\hline $\begin{array}{l}\text { Smoke/toxic inhalation } \\
\quad(n=852 / 14 / 190 / 45 / 83 / 520)\end{array}$ & $0(0)$ & $0(0)$ & $0(0)$ & $0(0)$ & $5(1.0)$ & 0.63 \\
\hline $\begin{array}{l}\text { Transfusion-related acute lung injury } \\
\quad(n=852 / 14 / 190 / 45 / 83 / 520)\end{array}$ & $1(7.1)$ & $10(5.3)$ & $2(4.4)$ & $3(3.6)$ & $28(5.4)$ & 0.92 \\
\hline Pulmonary vasculitis ( $n=852 / 14 / 190 / 45 / 83 / 520$ ) & $0(0)$ & $1(0.5)$ & $0(0)$ & $0(0)$ & $5(1.0)$ & 1 \\
\hline Drowning $(n=851 / 14 / 190 / 45 / 82 / 520)$ & $0(0)$ & $0(0)$ & $0(0)$ & $0(0)$ & $1(0.2)$ & 1 \\
\hline Other $(n=851 / 14 / 190 / 45 / 83 / 519)$ & $0(0)$ & $21(11.1)$ & $11(24.4)$ & $9(10.8)$ & $49(9.4)$ & 0.04 \\
\hline $\begin{array}{l}\text { Number of causes of hypoxemia } \\
(n=853 / 14 / 190 / 45 / 83 / 521)\end{array}$ & $1[0-1]$ & $1[1,2]$ & $2[1,2]$ & $2[1-3]$ & $2[1-3]$ & $<0.001$ \\
\hline $\begin{array}{l}\text { Number of causes of hypoxemia } \\
(n=853 / 14 / 190 / 45 / 83 / 521)\end{array}$ & & & & & & $<0.001$ \\
\hline None & $6(42.9)$ & $41(21.6)$ & $0(0)$ & $6(7.2)$ & $54(10.4)$ & \\
\hline 1 & $5(35.7)$ & $63(33.1)$ & $16(35.6)$ & $24(28.9)$ & $122(23.4)$ & \\
\hline 2 & $2(14.3)$ & $45(23.7)$ & $18(40.0)$ & $23(27.7)$ & $133(25.5)$ & \\
\hline 3 or more & $1(7.1)$ & $41(21.6)$ & $11(24.4)$ & $30(36.2)$ & $212(40.7)$ & \\
\hline Radiological infiltrates $(n=845 / 14 / 189 / 45 / 82 / 515)$ & $4(28.6)$ & $101(53.4)$ & $37(82.2)$ & $57(69.5)$ & $384(74.6)$ & $<0.001$ \\
\hline Unilateral c ( $n=583 / 4 / 101 / 37 / 56 / 385)$ & $1(25.0)$ & $36(35.6)$ & $9(24.3)$ & $19(33.9)$ & $111(28.8)$ & 0.58 \\
\hline Bilateral c $(n=581 / 4 / 100 / 37 / 56 / 384)$ & $3(75.0)$ & $65(65.0)$ & $28(75.7)$ & $37(66.1)$ & $273(71.1)$ & 0.65 \\
\hline ARDS & $0(0)$ & $0(0)$ & $0(0)$ & $9(10.7)$ & $169(32.2)$ & $<0.001$ \\
\hline Hypoxemia class & & & & & & $<0.001$ \\
\hline Mild & $11(78.6)$ & $136(71.2)$ & $11(24.4)$ & $43(51.2)$ & $239(45.5)$ & \\
\hline Moderate & $3(21.4)$ & $55(28.8)$ & $23(51.2)$ & $33(39.3)$ & $231(44.0)$ & \\
\hline Severe & $0(0)$ & $0(0)$ & $11(24.4)$ & $8(9.5)$ & $55(10.5)$ & \\
\hline $\begin{array}{l}\text { ICU length of stay (days) } \\
\quad(n=840 / 14 / 190 / 45 / 83 / 508)\end{array}$ & $11[4-21]$ & $8[4-17]$ & 14 [8-23] & $9[4-18]$ & $21[12-40]$ & $<0.001$ \\
\hline $\begin{array}{l}\text { ICU length of stay (days) in ICU survivors } \\
(n=625 / 11 / 170 / 37 / 70 / 337)\end{array}$ & $11[4-16]$ & $7.5[4-15]$ & $13[8-22]$ & $9[4-18]$ & $24[12-43]$ & $<0.001$ \\
\hline ICU mortality, N (\%) $(n=839 / 14 / 190 / 44 / 83 / 508)$ & $3(21.4)$ & $20(10.5)$ & $7(15.9)$ & $13(15.7)$ & $168(33.1)$ & $<0.001$ \\
\hline
\end{tabular}

than half of the patients hospitalized in ICUs the day of the study were hypoxemic, $79 \%$ did not fulfill the criteria of ARDS according to the Berlin definition [10]. The prevalence of ARDS, albeit greater in the most hypoxemic patients, was still less than half (42\%). Our results highlight also that hypoxemia is frequent among non-ventilated patients. The other main result of our study is that among hypoxemic patients, moderate and severe hypoxemia compared to mild hypoxemia was independently associated with higher ICU mortality.

Data concerning hypoxemia in unselected ICU patients, regardless of the presence of ARDS or the use 


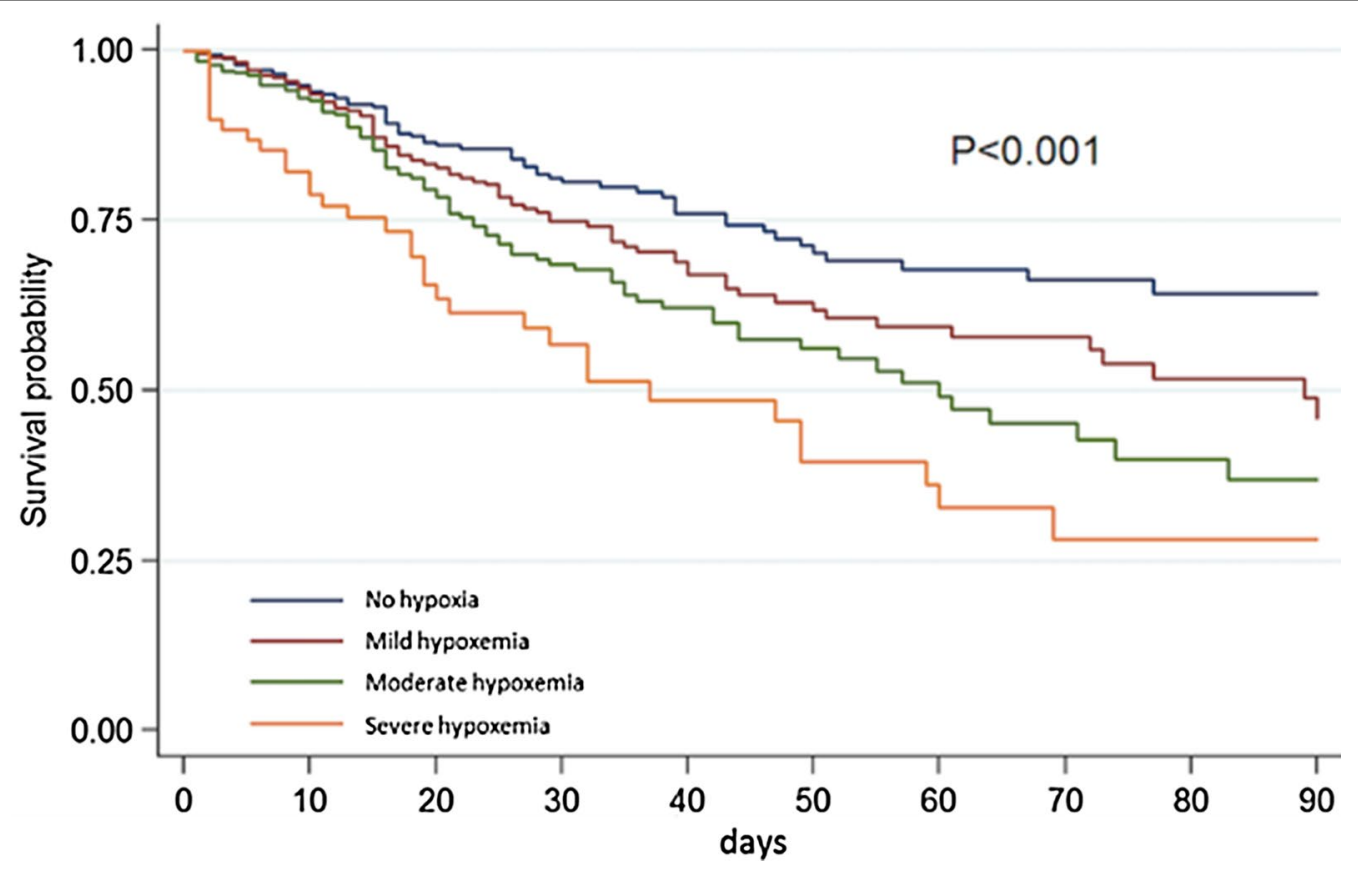

Fig. 2 Survival curve according to hypoxemia severity. Survival curves were drawn according to the severity of hypoxemia using the Kaplan-Meier method and were compared using the log-rank test. Follow-up ended at the ICU leaving or was censored at day 90

Table 5 Multivariate analysis of parameters associated with ICU survival

\begin{tabular}{|c|c|c|}
\hline & Adjusted HR x [95\% Cl] & $p$ \\
\hline Age & $1.01[1.00-1.02]$ & 0.054 \\
\hline Obesity & $0.63[0.45-0.89]$ & 0.008 \\
\hline Main diagnosis at ICU admission & & 0.049 \\
\hline Septic shock & $1.05[0.68-1.64]$ & \\
\hline Other shock & $1.83[1.09-3.07]$ & \\
\hline Severe trauma & $0.29[0.04-2.13]$ & \\
\hline De novo acute respiratory failure & 1 (ref) & \\
\hline Acute on chronic respiratory failure & $1.56[0.95-2.57]$ & \\
\hline Coma/seizures & $1.58[0.89-2.78]$ & \\
\hline Metabolic disorders & $0.74[0.25-2.19]$ & \\
\hline Cardiac arrest & $1.73[0.88-3.40]$ & \\
\hline Postoperative surveillance & $0.67[0.32-1.40]$ & \\
\hline Other & $0.76[0.37-1.56]$ & \\
\hline Chronic heart failure & $1.69[1.19-2.42]$ & 0.004 \\
\hline Chronic kidney failure & $1.65[1.05-2.62]$ & 0.032 \\
\hline Cirrhosis & $1.71[0.95-3.10]$ & 0.075 \\
\hline SAPS II (per point) & $1.01[1.00-1.02]$ & 0.012 \\
\hline Hypoxemia class & & $<0.001$ \\
\hline Mild & 1 (ref) & \\
\hline Moderate & $1.38[1.00-1.90]$ & \\
\hline Severe & $2.65[1.69-4.15]$ & \\
\hline
\end{tabular}

SAPS II Simplified Acute Physiology Score

Multivariate Cox analysis of variables associated with ICU survival of mechanical ventilation, are rather scarce, and we think this is strength of the SPECTRUM study to have included patients with all types of oxygenation devices. In the LUNG-SAFE study, hypoxemia was less frequent (35\%), but all the patients considered in the analysis received ventilatory support [4], unlike those analyzed in our study. In another study focusing on ARDS [5], the incidence of hypoxemia was 50\% among ventilated patients, but no data on non-invasively ventilated patients were given.

The prevalence of ARDS in the SPECTRUM study was $21 \%$ in hypoxemic patients, leading to an overall prevalence of $11 \%$ of included patients. This prevalence rate is close to the incidence found in the LUNG-SAFE study, in which $10.4 \%$ of the patients admitted in the ICU ( $23 \%$ of the mechanically ventilated patients) met the criteria for ARDS. Other studies, using the 1994 ALI/ARDS definition, reported similar results [5] or higher incidence $[7,8]$.

The presence of hypoxemia was associated with higher mortality and ICU length of stay, and the association with mortality was even stronger in the group of patients with severe hypoxemia. Severe hypoxemia was still associated with mortality after adjustment on respiratory support and in several sensitivity analyses, strengthening this result. This association is not so straightforward as several interventions known to improve $\mathrm{PaO}_{2} / \mathrm{FiO}_{2}$ 
ratio failed to reduce mortality [14] or were even detrimental $[15,16]$, suggesting that improving $\mathrm{PaO}_{2} / \mathrm{FiO}_{2}$ ratio cannot be an isolated therapeutic goal. Interestingly, ICU mortality among patients with mild-to-moderate hypoxemia was lower than that reported in patients with mild-to-moderate ARDS [4]. Conversely, mortality in our severely hypoxemic patients was close to that of severe ARDS patients described in other large studies. This result may be due to the fact that ARDS accounted for almost half of the severely hypoxemic patients in our cohort but also to the fact that similar pathophysiological mechanisms may be involved in severe hypoxemic patients with or without ARDS. For instance, pneumonia and aspiration as a cause of hypoxemia were more frequent in the most severe patients compared to other frequent causes of hypoxemia (see Table 2). Further studies should try to decipher the respective roles of ARDS and hypoxemia in the prognosis.

Invasiveness of respiratory support was also associated with mortality in univariate analysis, but time-dependent multivariate analysis did not confirm this association. Indeed, roles of respiratory support and severity of hypoxemia in mortality have a complex interplay as the most severe patients received the more invasive support and as respiratory support is not fixed across disease course. Our data suggest that severity of hypoxemia assessed by the $P / F$ ratio a given day is associated with mortality whatever the ventilator support needed in line with other study [17]. This finding may be useful in clinical practice to assess even roughly the severity of hypoxemia and the potential impact on outcomes.

We also evaluated the oxygenation devices used and the ventilator settings in hypoxemic patients. High-flow oxygen and NIV were not rare, even among severely hypoxemic patients. Almost $20 \%$ of the patients with a $\mathrm{PaO}_{2} / \mathrm{FiO}_{2}$ ratio $\leq 100$ were not on invasive mechanical ventilation, despite the fact that several studies have shown that NIV for de novo hypoxemic respiratory failure is associated with a poorer outcome in patients who finally required invasive mechanical ventilation [18-20]. We included all patients in the ICU and presenting hypoxemia (both de novo acute respiratory failure and acute on chronic respiratory failure), and it may be that those patients with severe hypoxemia on NIV had severe chronic respiratory failure or severe cardiogenic pulmonary edema. High-flow oxygen therapy was recently shown to have beneficial effects in patients with a $\mathrm{PaO}_{2} /$ $\mathrm{FiO}_{2}$ ratio $\leq 200$, as compared with standard oxygen and NIV in a randomized controlled trial [11]. However, the outcome of patients with the most severe hypoxemia receiving high-flow oxygen is unknown. Interestingly, in our study, high-flow oxygen was proportionally more frequently used in the most severely ill patients.
In mechanically ventilated patients, hypoxemia was associated with the use of "protective ventilation" in most cases with low $V_{\mathrm{t}}$ and higher PEEP, permissive hypercapnia and hypoxemia resulting in median plateau and driving pressures below the commonly admitted thresholds. Whereas reduced tidal volume appeared beneficial in observational studies among non-ARDS patients [21] and is currently the object of ongoing trials, the value of other protective strategies such as reducing driving pressure, increasing PEEP, or prone positioning may deserve evaluation in this population. The use of adjunctive therapies was correlated with the degree of hypoxemia. Neuromuscular blocking agents were the most often used in line with recent ARDS guidelines [22]: half of the patients with a $\mathrm{PaO}_{2} / \mathrm{FiO}_{2}$ ratio $\leq 100$ were continuously paralyzed. More surprising is the limited use of prone positioning on the day of the study in the subgroup of severely hypoxemic patients, even if not all patients fulfilled ARDS criteria. Recourse to prone positioning was, for instance, less frequent than the use of inhaled NO, whereas the level of evidence widely favors prone positioning rather than inhaled NO during ARDS [23-25].

Our study provides novel data about hypoxemia prevalence and management that could have implications for research and clinical practice. We provide an accurate prevalence of hypoxemia and stratification according to its severity even in those breathing spontaneously. This could help in the design of future interventional studies targeting these patients. In the clinical practice, physician should be alerted by the severity of hypoxemic non-intubated patients and the risk of mortality.

As we enrolled more than $90 \%$ of the screened patients, our observations were likely unbiased. However, our study has some limits. First, it was a point prevalence study and thus does not yield accurate data on incidence. By design, patients with prolonged stay had more chance to be included and are thus overrepresented in our study. Data on length of stay had then to be interpreted with caution and does not reflect accurately the mean length of stay of patients admitted in the ICU. Given the higher ICU length of stay of hypoxemic patients, one could hypothesize that the incidence of hypoxemia is lower than the prevalence. Conversely, some patients without hypoxemia on the day of study may have had hypoxemia before or after the study day during their ICU stay, and some hypoxemic patients may have been more severe. Likewise, our data on adjuvant therapies should be analyzed with caution as some, such as extracorporeal membrane oxygenation, cover a long period of time and so increase the chance of being present on the day of the study. Second, arterial blood gas was not analyzed in 31\% of patients. In those cases, hypoxemia was assessed with an extrapolation of the $\mathrm{PaO}_{2} / \mathrm{FiO}_{2}$ ratio from the $\mathrm{SpO}_{2} /$ 
$\mathrm{FiO}_{2}$ ratio. However, most patients classified as "hypoxemic" underwent arterial blood gas analysis on the day of the SPECTRUM study. Moreover, published data show that $\mathrm{PaO}_{2} / \mathrm{FiO}_{2}$ can be accurately derived from the $\mathrm{SpO}_{2} /$ $\mathrm{FiO}_{2}$ ratio [12, 26, 27] and sensitivity analysis including only patients with arterial blood gases was consistent with our result on mortality. Third, we conducted our study in spring to avoid the inclusion during an influenza seasonal pandemic; thus, our results could have been different in other seasons. Fourth, the inclusion of patients with limitation of treatment could impact our results as invasive devices are less likely to be used and mortality is higher in these patients. However, this allows to report in an unbiased way our epidemiological data. Lastly, the difficulty of separating ARDS from non-ARDS in hypoxemic patients is well known. In our survey, the diagnosis of ARDS was based on the Berlin criteria given in the CRF, independently of the clinical judgment of the clinician. However, chest-X ray analysis was not independently assessed. Even though we found a percentage of ARDS similar to that found in other large cohorts, it has been shown that ARDS is largely underdiagnosed, especially in its mild forms [4]. Even in the era of the Berlin definition, ARDS continues to be underrecognized, especially because of concerns about the reliable interpretation of chest radiographs [28]. Finally, although we identified an independent association between hypoxemia and mortality, our study does not provide mechanistic explanations of this finding.

\section{Conclusion}

In this 1-day point prevalence study, more than half of the patients suffered from hypoxemia, of mostly mild-tomoderate severity. ARDS criteria were present the day of data collection in only one out of five patients and were far from capturing the spectrum of hypoxemia in the ICU setting. Non-invasive methods of oxygenation were largely used even in the most hypoxemic patients. Finally, among hypoxemic patients, moderate and severe hypoxemia were independently associated with ICU survival, as compared to mild hypoxemia.

\section{Additional file}

Additional file 1: Table S1. List of contributors; Table S2. Patients' characteristics according to the presence of hypoxemia; Table S3. ARDS exclusion criteria among patients with bilateral infiltrates; Table S4. ICU mortality according to ventilator support and hypoxemia class; Table S5. Univariate analysis of variables associated with mortality in hypoxemic patients; Table S6. aHRs of $\mathrm{PaO} 2$ / FiO2 banding in different sensitivity analyses; Figure S1. Evolution of oxygen support after the day of study.
Authors' contributions

Not applicable.

\section{Acknowledgements}

The authors are deeply indebted to the patients who agreed to participate and to all physicians who took care of them. We also thank Chantal Sevens, Florence Neels, and Mathieu Lloung for their assistance. Special thanks to all involved in the collection and analysis of the data: all CERC members, all investigators, SRLF presidents and secretaries (Additional file 1: Table S1).

Writing committee: Grimaldi D (1) Hraiech S (2) Boutin E (3) Lacherade JC (4) Boissier F (5) Pham T (6) Richard JC (7) Thille AW (8) Ehrmann S (9) Lascarrou JB (10) Aissaoui N (11)

1. Intensive Care Unit, Clinique Universitaire de Bruxelles, Erasme hospital, Université Libre de Bruxelles (ULB) Route de Lennik, 808 1070, Brussels, Belgium

2. Aix-Marseille University, APHM, URMITE UMR CNRS 7278, Hôpital Nord, Réanimation des Détresses Respiratoires et Infections Sévères, Marseille, France

3. APHP, hopital Henri-Mondor, department of public health and clinical research unit (URC Mondor), 94010 Creteil Cedex, France

4. Médecine Intensive Réanimation, Centre Hospitalier Départemental de la Vendée, La Roche sur Yon, France

5. Service de Réanimation médicale, CHU de Poitiers, Poitiers, France; INSERM CIC 1402 (ALIVE group), Université de Poitiers, Poitiers, France 6. Interdepartmental Division of Critical Care Medicine, University of Toronto, Canada; Keenan Research Centre for Biomedical Science, Li Ka Shing Knowledge Institute, St. Michael's Hospital, Toronto, Canada. Sorbonne Universités, UPMC Université Paris 06, Paris, France

7. Service de Réanimation Médicale, Hôpital De La Croix Rousse, Hospices Civils de Lyon, Lyon, France. Université de Lyon, Université LYON I, Lyon, France. CREATIS INSERM 1044 CNRS 5220, Villeurbanne, FRANCE

8. CHU de Poitiers, Réanimation Médicale, Poitiers, France; INSERM CIC 1402 ALIVE, Université de Poitiers, Poitiers, France

9. Médecine Intensive Réanimation, CHRU de Tours, Tours, France; Centre d'étude des pathologies respiratoires, INSERM U1100, Aérosolthérapie et biomédicaments à visée respiratoire, Faculté de médecine, Université François Rabelais, Tours, France

10. Medical Intensive Care Unit CHU de Nantes 30 Bd. Jean Monnet 44093 Nantes cedex 1

11. Assistance Publique-Hôpitaux de Paris (AP-HP); Hôpital Européen Georges Pompidou (HEGP), Department of Critical Care Unit, Paris, France; Université Paris-Descartes, Paris, France; Paris-Cardiovascular-Research-Center, INSERM U970, Paris, France

\section{Competing interests}

The authors declare that they have no competing interests.

\section{Availability of data and materials}

The dataset supporting the conclusions of this article is available by asking to the SRLF secretary secretariat@srlf.org.

Consent for publication

Not applicable.

Ethics approval and consent to participate

Approval by the ethics committees according to the laws of each participating country was obtained. Patients were not included if they or their next of kin refused to participate.

\section{Funding}

The study was funded by the French Intensive Care Society (Société de Réanimation de langue française: SRLF Trial Group).

\section{Publisher's Note}

Springer Nature remains neutral with regard to jurisdictional claims in published maps and institutional affiliations. 
Received: 6 March 2018 Accepted: 2 July 2018

Published online: 13 August 2018

\section{References}

1. Luhr OR, Antonsen K, Karlsson M, Aardal S, Thorsteinsson A, Frostell CG, et al. Incidence and mortality after acute respiratory failure and acute respiratory distress syndrome in Sweden, Denmark, and Iceland. Am J Respir Crit Care Med. 1999;159(6):1849-61.

2. Vincent J-L, Akça S, De Mendonça A, Haji-Michael P, Sprung C, Moreno R, et al. The epidemiology of acute respiratory failure in critically ill patients $(*)$. Chest. 2002;121(5):1602-9.

3. The FINNALI-Study Group, Linko R, Okkonen M, Pettilä V, Perttilä J, Parviainen I, et al. Acute respiratory failure in intensive care units. FINNALI: a prospective cohort study. Intensive Care Med. 2009;35(8):1352-61.

4. Bellani G, Laffey JG, Pham T, Fan E, Brochard L, Esteban A, et al. Epidemiology, patterns of care, and mortality for patients with acute respiratory distress syndrome in intensive care units in 50 countries. JAMA. 2016:315(8):788

5. Roupie E, Lepage E, Wysocki M, Fagon JY, Chastre J, Dreyfuss D, et al. Prevalence, etiologies and outcome of the acute respiratory distress syndrome among hypoxemic ventilated patients. SRLF Collaborative Group on Mechanical Ventilation. Société de Réanimation de Langue Française. Intensive Care Med. 1999;25(9):920-9.

6. Brun-Buisson C, Minelli C, Bertolini G, Brazzi L, Pimentel J, Lewandowski $\mathrm{K}$, et al. Epidemiology and outcome of acute lung injury in European intensive care units. Results from the ALIVE study. Intensive Care Med. 2004;30(1):51-61.

7. Rubenfeld GD, Caldwell E, Peabody E, Weaver J, Martin DP, Neff M, et al. Incidence and outcomes of acute lung injury. N Engl J Med. 2005;353(16):1685-93.

8. Group TICCT. Acute lung injury and the acute respiratory distress syndrome in Ireland: a prospective audit of epidemiology and management. Crit Care. 2008:12(1):30.

9. on behalf of the ALIEN Network, Villar J, Blanco J, Añón JM, Santos-Bouza A, Blanch L, et al. The ALIEN study: incidence and outcome of acute respiratory distress syndrome in the era of lung protective ventilation. Intensive Care Med. 2011;37(12):1932-41.

10. ARDS Definition Task Force, Ranieri VM, Rubenfeld GD, Thompson BT, Ferguson ND, Caldwell E, Fan E, Camporota L, Slutsky AS. Acute respiratory distress syndrome: the Berlin definition. JAMA [Internet]. 2012 Jun 20 [cited 2017 Oct 6];307(23). Available from: http://jama.jamanetwork.com/ article.aspx?doi=10.1001/jama.2012.5669.

11. Frat J-P, Thille AW, Mercat A, Girault C, Ragot S, Perbet S, et al. High-flow oxygen through nasal cannula in acute hypoxemic respiratory failure. $N$ Engl J Med. 2015;372(23):2185-96.

12. Rice TW, Wheeler AP, Bernard GR, Hayden DL, Schoenfeld DA, Ware LB. Comparison of the $\mathrm{SpO}_{2} / \mathrm{FiO}_{2}$ ratio and the $\mathrm{PaO}_{2} / \mathrm{FiO}_{2}$ ratio in patients with acute lung injury or ARDS. Chest. 2007;132(2):410-7.

13. Amato MBP, Meade MO, Slutsky AS, Brochard L, Costa ELV, Schoenfeld $D A$, et al. Driving pressure and survival in the acute respiratory distress syndrome. N Engl J Med. 2015;372(8):747-55.
14. Taylor RW. Low-dose inhaled nitric oxide in patients with acute lung injury: a randomized controlled trial. JAMA. 2004;291(13):1603.

15. Acute Respiratory Distress Syndrome Network. Ventilation with lower tidal volumes as compared with traditional tidal volumes for acute lung injury and the acute respiratory distress syndrome. N Engl J Med. 2000;342(18):1301-8.

16. Derdak S, Mehta S, Stewart TE, Smith T, Rogers M, Buchman TG, et al. High-frequency oscillatory ventilation for acute respiratory distress syndrome in adults: a randomized, controlled trial. Am J Respir Crit Care Med. 2002;166(6):801-8.

17. Coudroy R, Frat J-P, Boissier F, Contou D, Robert R, Thille AW. Early identification of acute respiratory distress syndrome in the absence of positive pressure ventilation: implications for revision of the Berlin criteria for acute respiratory distress syndrome. Crit Care Med. 2018;46(4):540-6.

18. Demoule A, Girou E, Richard J-C, Taille S, Brochard L. Benefits and risks of success or failure of noninvasive ventilation. Intensive Care Med. 2006;32(11):1756-65.

19. Thille AW, Contou D, Fragnoli C, Córdoba-Izquierdo A, Boissier F, BrunBuisson C. Non-invasive ventilation for acute hypoxemic respiratory failure: intubation rate and risk factors. Crit Care. 2013;17(6):R269.

20. Bellani G, Laffey JG, Pham T, Madotto F, Fan E, Brochard L, et al. Noninvasive ventilation of patients with acute respiratory distress syndrome. Insights from the LUNG SAFE Study. Am J Respir Crit Care Med. 2017:195(1):67-77.

21. Serpa Neto A, Cardoso SO, Manetta JA, Pereira VGM, Espósito DC, Pasqualucci MDOP, et al. Association between use of lung-protective ventilation with lower tidal volumes and clinical outcomes among patients without acute respiratory distress syndrome: a meta-analysis. JAMA. 2012;308(16):1651.

22. Murray MJ, DeBlock H, Erstad B, Gray A, Jacobi J, Jordan C, et al. Clinical practice guidelines for sustained neuromuscular blockade in the adult critically ill patient. Crit Care Med. 2016;44(11):2079-103.

23. Guérin C, Reignier J, Richard J-C, Beuret P, Gacouin A, Boulain T, et al. Prone positioning in severe acute respiratory distress syndrome. N Engl J Med. 2013:368(23):2159-68.

24. Adhikari NKJ, Burns KEA, Friedrich JO, Granton JT, Cook DJ, Meade MO Effect of nitric oxide on oxygenation and mortality in acute lung injury: systematic review and meta-analysis. BMJ. 2007;334(7597):779.

25. Ruan S-Y, Wu H-Y, Lin H-H, Wu H-D, Yu C-J, Lai M-S. Inhaled nitric oxide and the risk of renal dysfunction in patients with acute respiratory distress syndrome: a propensity-matched cohort study. Crit Care [Internet]. 2016 Dec [cited 2017 Oct 6];20(1). Available from: http://ccforum.biomedcent ral.com/articles/10.1186/s13054-016-1566-0.

26. Ellis RK. Determination of $\mathrm{PO}_{2}$ from saturation. J Appl Physiol (Bethesda Md 1985). 1989;67(2):902.

27. Sanz F, Dean N, Dickerson J, Jones B, Knox D, Fernández-Fabrellas E, et al. Accuracy of $\mathrm{PaO}_{2} / \mathrm{FiO}_{2}$ calculated from $\mathrm{SpO}_{2}$ for severity assessment in ED patients with pneumonia: $\mathrm{PaO}_{2} / \mathrm{FiO}_{2}$ from $\mathrm{SpO}_{2}$ in pneumonia. Respirology. 2015;20(5):813-8.

28. Laffey JG, Pham T, Bellani G. Continued under-recognition of acute respiratory distress syndrome after the Berlin definition: what is the solution? Curr Opin Crit Care. 2017;23(1):10-7. 